\title{
Vrai « Faux Journal » de Jude Stéfan
}

\section{Marie-Françoise Lemonnier-Delpy}

\section{OpenEdition}

\section{Journals}

Édition électronique

URL : http://journals.openedition.org/genesis/474

DOI : $10.4000 /$ genesis. 474

ISSN : 2268-1590

\section{Éditeur :}

Presses universitaires de Paris Sorbonne (PUPS), Société internationale de génétique artistique littéraire et scientifique (SIGALES)

\section{Édition imprimée}

Date de publication : 1 janvier 2011

Pagination : 85-96

ISBN : 978-2-84050-749-9

ISSN : 1167-5101

\section{Référence électronique}

Marie-Françoise Lemonnier-Delpy, « Vrai «Faux Journal » de Jude Stéfan », Genesis [En ligne], 32 | 2011, mis en ligne le 18 septembre 2012, consulté le 20 avril 2019. URL : http:// journals.openedition.org/genesis/474; DOI : 10.4000/genesis.474 


\title{
Vrai « Faux Journal » de Jude Stéfan
}

\author{
Marie-Françoise Lemonnier-Delpy
}

$\mathrm{E}^{n}$ n octobre 1975 , la $N R F$ publie un volume de Journaux intimes inédits - soit un siècle d'écriture diaristique, puisque le recueil commence avec un texte de 1888 et se poursuit avec des extraits de journaux, écrits tout au long du $\mathrm{XX}^{\mathrm{e}}$ siècle jusque dans les années soixante-dix (1974 pour les fragments datés). L'avantdernier de la série est signé de Jude Stéfan et s'intitule «Faux Journal (ou : Une vie de bran) ». Il offre une double caractéristique. Il est publié dans un collectif où se retrouvent des textes déjà existants ou livrés par leurs auteurs pour figurer dans ce panorama de « journaux intimes et carnets » du $\mathrm{xx}^{\mathrm{e}}$ siècle et il n'a fait l'objet d'aucune publication autonome par la suite. Mais il marque le début d'une série d'œuvres stéfaniennes à caractère diaristique. Onze ans plus tard en effet, Jude Stéfan reprend le même titre de Faux Journal pour un livre qu'il publie seul. Si ce texte n'est pas la reprise de celui de 1975, il n'en constitue pas davantage la suite. Plusieurs aspects séparent les deux «Faux Journal ». Le premier, de 1975, ne mentionne aucune année de référence et répond à une sollicitation éditoriale de Gallimard. Le second, de 1986, porte sur les années 1979-1985 et est publié à l'initiative de Jude Stéfan1. Enfin, le texte de 1975 offre seul un intérêt tout particulier pour qui veut étudier la genèse du journal publié : il livre aujourd'hui une part de ses secrets, grâce au dépôt récent, par l'auteur, du tapuscrit sur lequel figurent des interventions auctoriales et surtout éditoriales.

Ce texte, publié par la $N R F$, mérite doublement son nom puisque, à la fausseté voulue par l'auteur et qui résonne comme une déclaration, une position de principe sur le genre diaristique, s'ajoute celle décidée par l'éditeur pour la publication. C'est en tout cas ce que prouve la confrontation entre d'une part le tapuscrit remis par l'auteur et revu par l'éditeur et d'autre part le texte publié dans la revue. Ce tapuscrit, resté inédit à ce jour dans son intégralité, donne à voir les coupures opérées dans le texte par l'éditeur. La question de la fausseté se trouve ainsi correspondre à deux démarches. La première, auctoriale, a pour spécificité d'être érigée en principe et exhibée par son titre, tandis que la seconde, éditoriale, est ignorée du lecteur et circonstancielle.

Jude Stéfan conçoit en effet la fausseté comme indissociable du journal. Initialement placée en soustitre dans le tapuscrit, la mention «Faux journal » était une sorte de commentaire générique du titre «Une vie de bran ». Le surgissement de l'écriture diaristique dans l'œuvre de Jude Stéfan s'accompagne alors d'une affirmation claire : les journaux intimes ou personnels sont des faux. Pour le «faux diariste », l'expression ellemême est pléonastique. Dans Dialogues avec la Sour qui paraît en 1986, soit la même année que Faux Journal, le deuxième du nom, il donne au lecteur le mode d'emploi du titre :

- Il faudrait lire le titre « Faux, journal », puisque le genre est fabriqué, composé, gauchi par lui-même, mais vrai : pas un miroir, ni un legs à la postérité $(\mathrm{sic})^{2}$

L'ordre choisi dans La Nouvelle Revue française place d'ailleurs à la fin cet exemple de contestation. Si l'on observe l'ensemble de ce numéro spécial, on remarque que certains textes ponctuent le volume comme

1. Nous avons étudié le texte publié de 1986 et ses suites dans l'article « Jude Stéfan, Faux journal », paru dans Métamorphoses du journal personnel, dir. Catherine Viollet et M.-F. Lemonnier-Delpy, Louvain-laNeuve, Academia-Bruylant, coll. "Au cœur des textes », 2006, p. 191-206. Nous y avons analysé précisément la notion de fausseté et en reprenons les éléments principaux. À l'époque, nous ignorions l'existence du tapuscrit d'auteur du texte de 1975, désormais propriété de la Médiathèque de Bernay.

2. Dialogues avec la Sæur, Seyssel, Éditions du Champ Vallon, 1987, p. 47. 
des réflexions théoriques sur le genre diaristique. Ils s'ajoutent aux analyses métadiscursives caractéristiques des journaux personnels. Ainsi la réflexion menée (p. 360) par Alain Clerval, "Les limites du journal intime », ouvre-t-elle le champ dans lequel le texte stéfanien trouve sa place (p. 389). En antépénultième position, celui-ci se situe au cœur d'un questionnement sur le genre. Il est par son thème centré sur la disparition, le frottement entre vie et mort. Le dernier texte, intitulé "L'adieu du père », de Christian Guidicelli en est une autre expression.

La démarche éditoriale a ses raisons. Jude Stéfan nous en a confié les modalités, lorsque nous l'avons, à plusieurs reprises, interrogé sur ce point. Le comité éditorial de la revue - Marcel Arland (rédacteur en chef), Dominique Aury et Jean Grosjean (secrétariat général) - se partage la relecture des articles. «Faux Journal » est, en l'occurrence, relu par Jean Grosjean. Celui-ci décide de supprimer un certain nombre d'entrées, sans en référer à l'auteur. Ce dernier, alors moins connu qu'aujourd'hui, ne remet pas en cause cette démarche et la liberté d'intervention de l'éditeur. Il reçoit une explication de ce choix éditorial : les suppressions ont été effectuées afin de ne pas entraîner de trop vives réactions des lecteurs abonnés à la revue. Cet argument donne la mesure du caractère à l'époque scandaleux de ce journal, moralement dérangeant par ses thématiques mais aussi susceptible de heurter la sensibilité d'un lectorat croyant, par la présence de notations ouvertement blasphématoires. D'ailleurs, après la parution, la réaction hostile de certains lecteurs confirme la lucidité de l'éditeur et peut légitimer la décision prise.

Le poète et diariste avait, à l'époque, souligné, sur la page de titre du tapuscrit, le caractère « tronqué » du texte (voir fig. 1). En lisant ce Faux Journal aujourd'hui, notre propos ne sera pas d'instruire le procès de l'éditeur mais bien, à la manière dont cela a pu être fait pour d'autres textes littéraires, de revenir aux fragments supprimés et d'observer ce qu'induit leur présence dans la version originale. II s'agit en quelque sorte de ressusciter une unité que voulut l'auteur en son temps et d'observer quelle sorte de journal elle donne à lire. La confrontation entre le tapuscrit déposé par l'auteur ${ }^{3}$ et le texte publié permet de saisir ce que le second laisse délibérément de côté.
Cette enquête suppose que soit prêtée la plus grande attention à l'ordre et au choix des entrées. Pour ce faire, après avoir présenté le tapuscrit dans sa matérialité et caractérisé les étapes des interventions auctoriales qui sont également présentes et significatives d'un projet d'écriture, nous proposerons une lecture du texte intégral. Cela nous conduira à rendre sensible ce que la version publiée a délaissé et qui déroule une véritable construction du «moi » à travers un parcours ordonné du temps, des états du corps, des autoportraits, le tout triomphant dans l'expulsion de soi. Si le texte édité préserve le mouvement qui conduit implacablement à l'épilogue du texte, l'agencement initial que donne à lire le tapuscrit confère une force incomparable à la construction de soi opérée par Jude Stéfan.

\section{Le tapuscrit, objet du sujet Jude Stéfan}

\section{Présentation matérielle du tapuscrit ${ }^{4}$}

En comprenant la page de titre, le tapuscrit compte dix-huit pages. Il est tapé par l'auteur sur deux papiers différents, l'un non filigrané, l'autre à filigrane (Montérain, extrastrong) à partir de la page 8 , sans que l'on puisse vraiment en déduire quoi que ce soit sur le calendrier d'écriture du texte. L'ensemble de ces dix-huit pages a subi une recomposition que trahissent numérotation et état du texte. En effet la pagination des folios est tantôt manuscrite, tantôt tapuscrite. Les treize premiers folios sont numérotés de la main de l'auteur de la manière suivante :

- en rouge de la page 1 à 7 ;

- en bleu pour les pages $8,9,10$;

- en rouge, page 11, sur un grattage page 12 ;

- en bleu et dans la marge, page 13, sur une feuille dont le bas a été coupé sur $5 \mathrm{~cm}$.

3. Les documents reproduits ici le sont avec l'aimable autorisation de la Ville de Bernay et de Jude Stéfan. Nous leur adressons nos vifs remerciements.

4. L'ensemble de cette étude renvoie au tableau ci-dessous. Nous avons numéroté les entrées des deux textes que nous comparons, le tapuscrit et le texte publié. Les numéros figurant entre parenthèses dans cet article sont ceux du tapuscrit. 


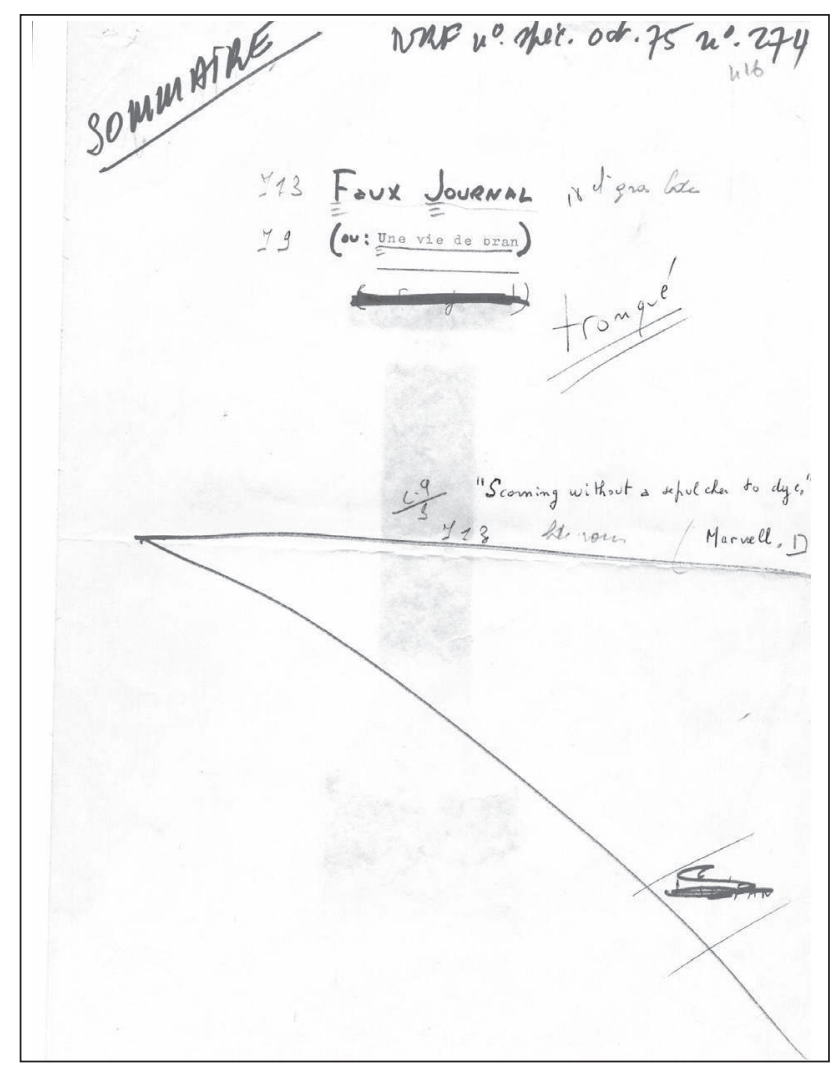

Fig. 1 : Page de titre du tapuscrit avec les modifications de l'éditeur (le sous-titre devient titre)

et l'appréciation de l'auteur soulignée en bleu

Les folios 14 et 15 offrent une numérotation tapuscrite rouge, tandis que les suivants (16 et 17) portent des numéros inscrits au stylo-bille rouge.

Enfin la recomposition est explicite dans la numérotation du folio 5 surajouté et surtout du 6 nommé $6^{2}$. On comprend qu'il a dû exister une page 5, absente ou renumérotée. De même la page 13 est amputée par l'auteur et les pages 14 et 15 ont dû être écrites à la suite après un remaniement puisque la phrase du feuillet 15 (pagination tapuscrite) se poursuit feuillet 16 (pagination manuscrite) sans qu'il y ait rupture. Ainsi ces irrégularités dans la typographie de la numérotation sont l'indice qu'il y a eu sur ce tapuscrit plusieurs campagnes d'écriture. Il y en a d'ailleurs eu très certainement d'autres auparavant mais, en règle générale,
Jude Stéfan jette les versions transformées de ces textes. L'observation des corrections présentes ici nous convainc cependant qu'il y en a trois identifiables.

\section{Observation matérielle des corrections}

Le texte est tapé à l'encre rouge (comme nombre d'autres tapuscrits de Jude Stéfan). Les corrections, commentaires et ajouts de l'auteur sont tracés au stylobille tantôt rouge, tantôt bleu. Cette dualité apparaît dans le tableau ci-dessus qui présente l'état du texte en ses multiples aspects. On y observe qu'une même correction peut offrir deux strates : biffure en bleu et correction en rouge. À une occasion, la réécriture juxtapose deux encres bleues $(39$, p. 16).

Quand ces reprises de son texte par l'auteur se situent-elles ? Certaines ont lieu à l'évidence au cours du processus d'écriture puisque la correction, faite soit à la machine soit au stylo rouge, détermine la suite immédiate du propos. D'autres semblent postérieures et offrent une biffure et réécriture des termes à modifier. Ces interventions auctoriales se distinguent de celles de l'éditeur, réalisées au feutre rouge et au crayon à papier.

On peut donc schématiser les diverses interventions comme suit :

1. Interventions auctoriales de correction saisie au cours de la dactylographie (ex : 36, p. 14, signe $=<$ le juste signe > ; 38, p. 16, ridieule <grossier>).

2. Interventions auctoriales de correction, correction manuscrite sur le tapuscrit (ex : 4, p. $3 ; 6$, p. 4) avec ou sans ajout. Ces modifications se déclinent de plusieurs façons :

- suppression sans ajout

- correction en surimpression sur le mot ou texte tapé

- biffure puis ajout interlinéaire (ex : 6, p. 4, mais <or>)

- ajout manuscrit soit d'un mot qui manque (ex : 22, p. 10 , ajout de « cesse » après « sans »), soit d'un texte nouveau (ex : 28 , p. $12 ; 32$, p. $13 ; 37$, p. $15 ; 39$, p. 16).

3. Interventions éditoriales.

4. Commentaire auctorial sur la page de titre : «Tronqué » écrit en bleu et souligné deux fois par l'auteur, commentaire après-coup sur le texte transformé par l'éditeur. 


\begin{tabular}{|c|c|c|c|c|c|}
\hline $\begin{array}{l}\text { Pagination } \\
\text { du tapuscrit } \\
\text { (manuscrite } \\
\text { ou } \\
\text { tapuscrite) }\end{array}$ & $\begin{array}{l}\text { Entrées du tapuscrit avec } \\
\text { ajouts manuscrits de l'auteur } \\
\text { (en italique) et corrections } \\
\text { éditoriales }\end{array}$ & $\begin{array}{l}\text { Modifications } \\
\text { (bleu) }\end{array}$ & $\begin{array}{l}\text { Modifications } \\
\text { (rouge) }\end{array}$ & $\begin{array}{l}\text { Pagination } \\
\text { du texte } \\
\text { édité }\end{array}$ & Entrées du texte édité \\
\hline Page de titre & $\begin{array}{l}\text { Faux Journal ou Une vie de } \\
\text { bran (ajout du titre + inversion } \\
\text { titre/ss-titre par l'éditeur) }\end{array}$ & $\begin{array}{l}\text { Tronqué } \\
\text { Épigraphe }\end{array}$ & & & $\begin{array}{l}\text { Titre, sous-titre, } \\
\text { épigraphe }\end{array}$ \\
\hline \multirow[t]{2}{*}{ p. 1 (man.) } & 1/ 1er janvier & & & p. 389 & 1/ 1er janvier \\
\hline & 2/ Nouvelle lune & & 1 cor. & & 2/ Nouvelle lune \\
\hline p. 2 & 3/ Jour du Seigneur, le 17 & & & p. 390 & 3/ Jour du Seigneur, le 17 \\
\hline p. $2-3$ & 4/ Sainte Prisca & 2 cor. & & p. $390-391$ & 4/ Sainte Prisca \\
\hline p. 3 & 5/ Jour de Vénus 26 & & & & 5/ Jour de Vénus 26 \\
\hline p. 4 & $\begin{array}{l}\text { 6/ Le } 16 \text { de ce mois des } \\
\text { Purifications ou février }\end{array}$ & 2 cor. & & p. 392 & $\begin{array}{l}\text { 6/ Le } 16 \text { de ce mois des } \\
\text { Purifications ou février }\end{array}$ \\
\hline p. $4-5$ & 7/ Jour de grâce 18 & & & p. $392-393$ & 7/ Jour de grâce 18 \\
\hline p. $5(\operatorname{sur} 6)$ & $8 / 20$ ou 21 & & 1 cor. & p. 393 & $8 / 20$ ou 21 \\
\hline $\begin{array}{l}\text { p. } 5(\operatorname{sur} 6)- \\
62\end{array}$ & $\begin{array}{l}\text { 9/Tel février de l'inexplicable } \\
\text { présence }\end{array}$ & & & p. 393-394 & $\begin{array}{l}\text { 9/ Tel février de } \\
\text { l'inexplicable présence }\end{array}$ \\
\hline p. $6^{2}-7$ & 10/ Fin février & & 2 cor. & Supprimé & \\
\hline p. 7 & 11/ Ier Mars & & 2 aj. & Supprimé & \\
\hline p. 7 & 12/ 11 Pluviôse & & & Supprimé & \\
\hline p. 7 & 13/ lundi 12 mars & & & p. 393-394 & 10/ lundi 12 mars \\
\hline p. 8 & 14/ Même date & 1 cor. & & Supprimé & \\
\hline p. 8 & $\begin{array}{l}15 / \text { Ier de l'ouverture des saisons } \\
\text { ou avril }\end{array}$ & & & p. 395 & $\begin{array}{l}11 / 1^{\mathrm{er}} \text { de l'ouverture des } \\
\text { saisons ou avril }\end{array}$ \\
\hline p. 9 & 16/ 13 Avril du printemps & & & Supprimé & \\
\hline p. 9 & 17/ 14. Pensée & & 1 sup. & Supprimé & \\
\hline p. 9 & $18 / 15$ & & 2 aj. & p. 395 & $12 / 15$ \\
\hline p. 9-10 & $19 / 16$. & & & Supprimé & \\
\hline p. 10 & $20 / 17$ & & & p. 395 & $13 / 17$ \\
\hline p. 10 & $21 / 18$. & & & p. 395 & $14 / 18$ \\
\hline p. 10 & $22 / 19$. & 1 cor., 1 aj. & 1 cor. & Supprimé & \\
\hline p. 10 & $23 / 20$ & & & Supprimé & \\
\hline p. 11 & 24/ Mémorable dimanche 23 & & 1 aj. & Supprimé & \\
\hline p. $11-12$ & 25/ Saint Fidèle & & & p. 396 & 15/ Saint Fidèle \\
\hline p. 12 & 26/ Chose vue le 29 & & & p. 396 & 16/ Chose vue le 29 \\
\hline p. 12 & 27/ Ier Mai & & & Supprimé & \\
\hline
\end{tabular}




\begin{tabular}{|c|c|c|c|c|c|}
\hline $\begin{array}{l}\text { Pagination } \\
\text { du tapuscrit } \\
\text { (manuscrite } \\
\text { ou } \\
\text { tapuscrite) }\end{array}$ & $\begin{array}{l}\text { Entrées du tapuscrit avec } \\
\text { ajouts manuscrits de l'auteur } \\
\text { (en italique) et corrections } \\
\text { éditoriales }\end{array}$ & $\begin{array}{l}\text { Modifications } \\
\text { (bleu) }\end{array}$ & $\begin{array}{l}\text { Modifications } \\
\text { (rouge) }\end{array}$ & $\begin{array}{l}\text { Pagination } \\
\text { du texte } \\
\text { édité }\end{array}$ & Entrées du texte édité \\
\hline p. 12 & $28 / 2 M a i$ & Tt ajouté & & p. 396 & 17/2 Mai \\
\hline p. 13 & 29/3 mai & & & p. 396-397 & 18/3 mai \\
\hline p. 13 & 30/ 4 mai & & & Supprimé & \\
\hline p. 13 & $31 / 15$. & & & p. 397 & $19 / 15$ \\
\hline p. 13 & $32 / 16$. & 1 aj. & 1 cor. & p. 397 & $20 / 16$ \\
\hline p. 13 & $33 / 17$ & & 1 aj. & p. 397 & $21 / 17$ \\
\hline p. 14 & $34 / 25$ mai & & & p. 397 & $22 / 25$ mai \\
\hline p. 14 & 35/ Dans le désert de juillet & & & Supprimé & \\
\hline p. 14 & 36/ en août & & 2 cor. & p. $397-398$ & $23 /$ en août \\
\hline p. 15 & 37/ En la paix des choses mortes & 1 aj., 1 cor. & & Supprimé & \\
\hline p. $15-16$ & 38/ Fin septembre & & & p. 398 & 24/ Fin septembre \\
\hline p. 16 & 39/ Ce 30 septembre & 1 cor., 1 aj. & $\begin{array}{l}1 \text { cor. } \\
1 \text { aj. }\end{array}$ & p. 398-399 & 25/ Ce 30 septembre \\
\hline p. 17 & 40/ 1er novembre & & & p. 399 & 26/1er novembre \\
\hline p. 17 & 41/ Fin novembre & & & p. $399-400$ & 27/ Fin novembre \\
\hline
\end{tabular}

Fig. 2 : Tableau comparatif du tapuscrit et du texte édité portant mention des ajouts et des modifications (en bleu et en rouge) de Jude Stéfan ainsi que des suppressions et des interventions de l'éditeur

\section{Ce que disent la recomposition et les corrections auctoriales...}

La recomposition confirme le parti pris esthétique de Jude Stéfan. «Faux Journal » il y a puisque ce texte est bien une réécriture du journal ou une composition, à partir de notes. S'affiche ainsi une rupture du pacte d'authenticité qui est associé à l'idée que le lecteur se fait du journal, produit, pense-t-il, d'une écriture souvent régulière et intouchée par le diariste. Mais en exhibant la fausseté dans son sous-titre 5 , Jude Stéfan respecte ce pacte, paradoxalement.

Les corrections auctoriales expriment souvent un souci d'exactitude, dans le choix des mots, mais inscrivent aussi le texte dans deux registres opposés. Le premier renvoie au prosaïsme et au petit fait vrai, le second opte pour la transposition quasi métaphysique du moment vécu. Il y a oscillation entre les deux, avec effet d'affirmation ou de rectification 6 de chacun de ces aspects. Or cette tension entre ces langages apparemment antagoniques reflète parfaitement la finalité de ce texte, telle qu'elle apparaît au lecteur, à réception.

Les prosaïsmes foisonnent : ils sont à la fois la réalité et le fil conducteur de ce texte : états du corps, nourriture, bulletins météorologiques définissent le quotidien. Ainsi, l'ajout du 30 septembre (39, p. 16), dans une parenthèse finale, décrit le sac mortuaire de la chienne : «(Ce sac mortuaire est un sac postal, qu'elle avait d'ailleurs flairé, à la livraison $\neq$ de dictionnaires) ».

5. Il y a eu inversion de l'ordre prévu par l'auteur (voir fig. 1 et 2). 6. Ainsi, la rectification d' « air mort » en « air moite » (9) transforme la notation existentielle ou métaphysique en notation climatique. 
La précision marque la fidélité au réel 7 , mais construit aussi un réseau de significations, en liant le sac à la poste auparavant évoquée et aux dictionnaires dont l'allusion jalonne le texte. Elle fait sens et permet de sortir du chagrin suggéré par les phrases nominales et laconiques qui précèdent cet ajout : «Dans un sac, dans un trou du jardin. Une prière pour elle. » Cette entrée du 30 septembre est d'ailleurs la seule à recourir à un effet que le texte publié ne reprend pas, le soulignement d'une expression. "Ce trente septembre » qui, par le démonstratif, nous plonge dans l'instant vécu, commence en effet par ces mots : "Je ne puis supporter de voir mourir la vie. La chienne a été prise de paralysie à l'arrière-train. »

Notations «métaphysiques », les ajouts manuscrits des vingt-huitième et trente-septième entrées disent explicitement le rapprochement permanent entre vie et mort. La première entrée, celle du 2 mai, « Ô Mort, grande vacance », et la conclusion de la trentesixième, "Ici sont les limbes », ne prennent sens que par rapport à ce qui les précède, respectivement l'entrée «1er mai. Excellentes selles » et l'entrée « En la paix des choses mortes » qui introduit un après-midi « À l'académie de billard » (voir fig. 3). On y retrouve l'ironie des rapprochements et le contraste des registres. Ces ajouts construisent le texte en ses effets cinglants et interrogateurs.

C'est bien ce couplage que le tapuscrit auctorial élabore, puis que le tapuscrit éditorial modifie.

\section{Jude Stéfan en ces autoportraits}

\section{Libre scansion du temps}

Reportons-nous au déroulé du tapuscrit d'auteur avant de le confronter au texte publié. Il est aisé d'y observer quarante et une entrées pour ce qui couvre une «presque » année. Le journal commence le 1 er janvier et s'achève « fin novembre ». Aucune mention d'année pour un faux journal qui propose un déroulement symbolique de dires et de faits. Des biographèmes disposés dans le texte permettent-ils de révéler une suite chronologique ? Oui, mais à condition de faire «parler» le texte, ce que rien ne prescrit. On suppose en effet que le journal retient ce que le diariste souhaite consigner (ainsi il n'a guère besoin de s'expliquer à lui-même ses allusions personnelles), sans souci de ce qui est nécessaire à un tiers, lecteur, à moins que le journal ne soit adressé. Non, si l'on est attentif à la reconstruction que révèle Faux Journal.

Prêtons-nous néanmoins à l'exercice. Cela reviendrait par exemple à comprendre la mention du « divorce de ma femme aimée » dans l'entrée 13 (reprise dans l'entrée 14) comme la séparation dans les faits de Jude Stéfan « d'avec sa femme Édith » selon les termes d'une notice biographique autographe, postérieure à la parution de ce texte, qui mentionne l'an 1974. Or aucun prénom, par exemple, ne nous y autorise clairement. Tout aussi importante, la mort de la chienne que nous évoquions il y a peu, privée de nom elle aussi. Enfin, dernier exemple, l'« attaque rénale », évoquée dans la neuvième entrée, pourrait renvoyer à la décennie précédente, la « crise rénale » que mentionne « Annales ${ }^{8}$ » en son chapitre IV $\left(n^{\circ} 60\right)$. Il est remarquable que nous n'identifiions tous ces faits qu'en sortant du texte du Faux journal pour aller trouver ailleurs leur substance « biographique ».

Or ce n'est pas avec ce regard scrutateur que la lecture du Faux Journal prend sens. Tout juste nous révèle-t-elle que le texte résulte d'un entremêlement d'éléments réels choisis et rendus expressifs par leur place dans cette série de dates. L'enchaînement interne des entrées successives compterait davantage que le référent réel. Le rythme du Faux Journal s'offre dès lors comme un choix construit de fragments qui tracent une ou des identités au diariste.

Rythme donc. Ou plutôt rythmes.

S'arrêter fin novembre. Dans la suite de ses écrits diaristiques, Jude Stéfan délaisse souvent le mois de décembre. Ainsi dans Faux Journal, paru en 1986, sur les sept chapitres (1979-1985), décembre ${ }^{9}$ n'est là qu'en 1979 et 1980 puis il disparaît au même titre que d'autres mois. Le calendrier que forge « Faux Journal » de 1975 est aussi oublieux de juin et d'octobre.

7. La notation du 15 mai (31, p. 13), « et trois crêpes » ajoutée à « un jus de raisin », rompt la liste uniquement composée de boissons.

8. «Annales (ou fable stéfanienne) », Variété VII, Éditions Le temps qu'il fait, 1999, p. 11-15.

9. Pour Jude Stéfan, « l'année climatologique commence en mars, le premier temps, comme les Romains le fêtaient, loin des niaiseries papistes », Faux Journal, op. cit., 1986, p. 7. 


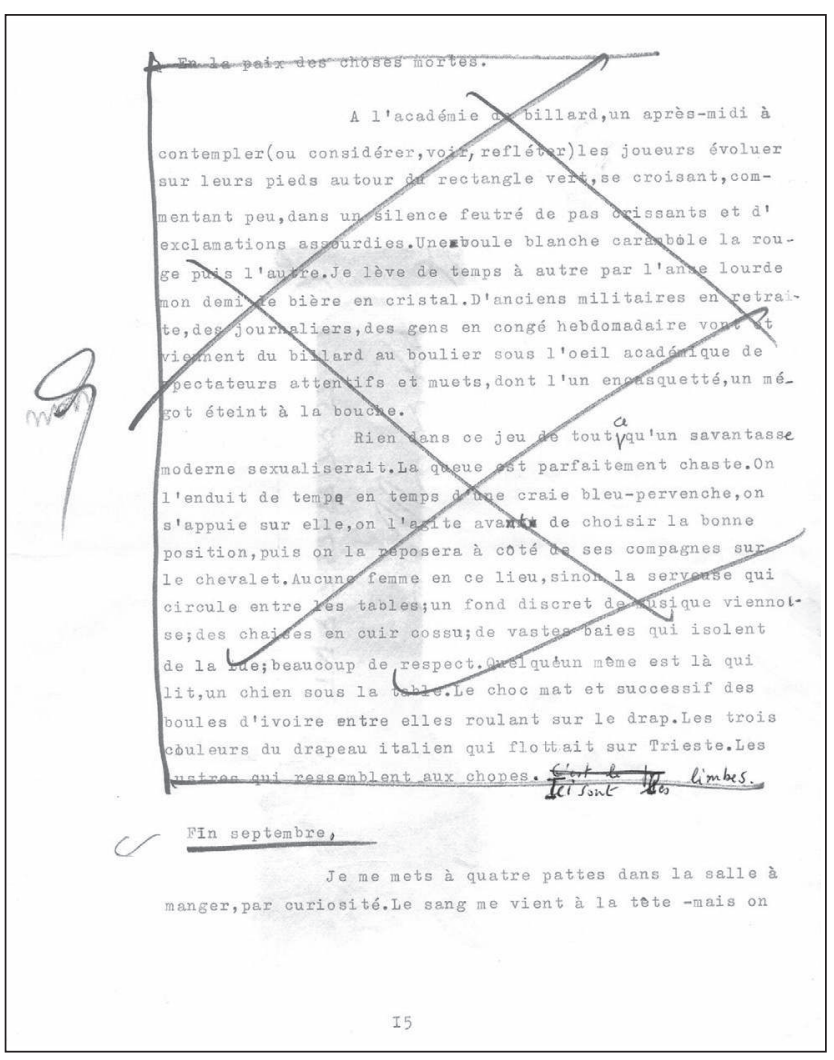

Fig. 3 : Entrée ${ }^{\circ} 37$ supprimée dans l'édition publiée, dont le titre « En la paix des choses mortes» et l'ajout manuscrit, en conclusion, de la formule « Ici sont les limbes », illustrent la dimension philosophique et la part d'invention du Faux Journal. Parmi les « spectateurs attentifs et muets », on reconnaît Jude Stéfan, « encasquetté », à la fin du premier paragraphe

Le texte de 1975 offre un cadre temporel à la fois apparemment banal et original. La banalité tient au fait que toutes les entrées, ou presque, lient le texte à une date identifiable comme on l'attend en règle générale du journal. Toutefois ces dates se rattachent à des univers de référence différents - calendriers chrétien et révolutionnaire, réactivation étymologique de la dénomination des jours de la semaine ${ }^{10}$ - qui brouillent les pistes de l'ordonnancement usuel des jours et des nuits tout en y renvoyant. À cette désynchronisation s'ajoute une arythmie : les mois comptent des entrées tantôt nombreuses, tantôt rares, tantôt uniques, tantôt absentes. Ce mélange des rythmes et des mesures du temps rejoint et annonce ce que l'œuvre stéfanienne montre par ailleurs. Par exemple, avec quarante et une entrées, ce texte-ci est annonciateur du Faux Journal de 1986 dont les chapitres-années I et II comptent une quarantaine d'entrées, tandis que la tendance est nettement à l'amenuisement, à la raréfaction des entrées par la suite, de 1981 à 1985.

Les jours se suivent et s'unissent ou se séparent de par leur titre. «Jour du Seigneur, le 17 » (3) précède de peu « jour de Vénus » (5) et tous deux trouvent un écho dans «Jour de grâce 18 » (7). Les saints de même : «Sainte Prisca » (4) annonce «Saint Fidèle » (25). Dans cette suite de jours, l'oscillation est présente (comme dans l'entrée intitulée « 20 ou 21 », 8) et contraste avec la mention spéciale qui accompagne certaines dates : «tel février de l'inexplicable présence » (9), « Mémorable dimanche 23 » (24). Le jour, sous la plume de Jude Stéfan est le seul moment d'écriture affirmé11. Ainsi la nuit faitelle l'objet d'un récit dans certaines entrées (3, 9, 23, 29,39 ) où elle est intimement associée à la sexualité, aux affres et bonheurs du sommeil et à la mort, ici celle de la chienne. L'entrée 32 «Lever : 8 h 27 ; coucher : 20 h 27, guilleret » s'offre comme le rêve de parfait équilibre des temps diurnes et nocturnes. Sans l'ajout manuscrit «guilleret», elle est comme le degré zéro du journal. Avec cette mention, elle note l'humeur du diariste, assortie à l'humoristique exactitude temporelle.

L'autre visage du temps auquel le diariste accorde une place est celui des variations météorologiques. Cet aspect concerne l'entrée 10, supprimée dans le texte publié :

\section{Fin février}

Ma vie météorologique. Toute journée y offre sa passionnante énigme. Fait-il trop élevé pour l'exécrable neige $\left(6^{\circ}\right)$ ? $\mathrm{La}^{*}$ brouillard va-t-il demeurer aussi extasiant et bas $\left(3^{\circ}\right)$ ? Un adoucissement se présage $\left(8^{\circ}\right)$. Une douce pluie nous protège du froid $\left(9^{\circ}\right)$. Il gèle, certes, mais il fera beau $\left(63^{\circ}\right)$ Hélas la saloperie de neige offusque nos lèvres $\left(1^{\circ}\right)$, une tempête nocturne nous éveille $\left(2^{\circ}\right)$, une bise venue de l'est nous scie les « jambes » $\left(6^{\circ}\right)$. Une averse de grêle heurte les toits $\left(4^{\circ}\right)$; le soleil revenu lave les champs de l'ordure blanche $\left(5^{\circ}\right)$; la bonne * $* x \times *$ rouvre la fenêtre au midi $\left(7^{\circ}\right) ; 1^{\prime}$ 'eau du caniveau est gelée ce matin $\left(0^{\circ}\right)$. Du grésil dans la campagne $\left(-2^{\circ}\right)$

10. Comme « Jour de Vénus » $\left(\mathrm{n}^{\circ} 5\right)$.

11. Il préfère d'ailleurs le mot « diurnal » à celui de « journal ». 
S'il en était besoin, ce passage nous rappelle l'importance du temps qu'il fait, celui que mesurent baromètre et thermomètre, instruments de précision mentionnés plus loin (35). Les premiers commentaires introduisant le catalogue des bulletins météorologiques possibles « fin février » confèrent à cette entrée une fonction synthétique en même temps qu'ils offrent des variations poétiques sur les intempéries. La volonté affirmée du diariste de ponctuer chaque note ou appréciation subjective d'indications thermiques précises objective et exemplifie l'autoportrait qu'est «Ma vie météorologique ». Plus largement, le cadre temporel qui se dessine tout au long des quarante et un fragments élus par le diariste constitue une composante de son autoportrait. Il a pour caractéristique de souligner une perception personnelle du temps tout en se démarquant poétiquement ou en usant ironiquement de ce que, dans l'entrée 5 (« Jour de Vénus $26 »)$, Jude Stéfan appelle « les inepties usuelles sur le temps ». À la suite du fragment 10 (« Fin février»), une autre entrée sacrifiée par l'éditeur - «1er mars » (11) - met en pleine lumière ce rapport aux mois :

1 er mars

Moi, Jude, affirme avoir salué en ce jour de ma frêtale main, la mort de l'infâme février, mon ennemi.

Je songe déjà à retirer mes sous-vêtements de flanelle « car je suis un héros », maintenant que la température avoisine les dix degrés et plus, de même je décide de changer bientôt de pull-over.

Ce solennel salut et adieu à la saison honnie théâtralise le journal et fait entendre une de ses voix - littéraire par le recours au pseudonyme Jude - tout en rappelant la thématique essentielle du vêtement dans ce texte de Jude Stéfan.

\section{Corps habillé, corps nu du journal}

Le journal, corps constitué, restructuré par la volonté de l'auteur comme en témoignent les traces de recomposition, puis par celle de l'éditeur comme le montrent les suppressions sur le tapuscrit, fait effectivement une large part aux «états du corps », pour reprendre le titre d'un recueil de nouvelles de Jude Stéfan.
Il s'ouvre d'ailleurs sur ce sujet puisque les premiers et derniers mots de la prime entrée lui sont consacrés :

$$
\text { 1er janvier }
$$

Je n'aime plus guère que ceci : me laver les pieds afin de les sentir dans des chaussettes propres - que je ne laisse à personne le soin de laver ; [...] ; déféquer régulièrement ; m'endormir en chien de fusil.

Non sans esprit de provocation, le journal affiche son parti pris de dérouler un quotidien centré sur la physiologie et la scatologie. De nombreuses notations expriment une attention aux pieds, aux fonctions intestinales en tout genre, aux accidents de santé, aux soins corporels, attention assortie à/de la présentation de lieux spécifiques comme les urinoirs et leurs graffitis, les hôpitaux et leurs pensionnaires, les salons de coiffure. Parmi ces entrées prosaïques, comble de la déréliction pour certains, simple état de fait pour Jude Stéfan, un bon nombre ont été supprimées par l'éditeur: entrées 18 du 16 (avril), 23 et 24 des 19 et 20, $30 \mathrm{du} 4$ mai, et enfin, entrée 35 sur les plaisirs de la « grattaison ». Or ces considérations forment une suite dans laquelle le diariste va au bout de l'expression d'un moi charnel et humoral. Insérée dans la trame du texte, cette suite est en harmonie avec l'omniprésente thématique du rien et du méprisable et nourrit l'autoportrait moral de Jude Stéfan.

\section{Autoportraits}

Comment cet autoportrait surgit-il dans le texte ? Il en est la toile de fond mais certaines entrées le font passer au premier plan en introduisant un véritable discours de présentation. La confession en est littéralement l'occasion. Pour la Sainte Prisca - dont le prénom évoque par son étymologie la vieillesse et par son histoire, la latinité voici le diariste-narrateur qui part à confesse : « ne sachant comment atteindre le sommeil, je vais dans une église me confesser ». Cette confession réintroduit la Stéfanie ${ }^{12}$, c'est-à-dire l'univers fictif que s'est forgé Jude Stéfan, ici en l'occurrence ses trois sœurs que l'on retrouve dans

12. Comme il existe une Stendhalie, il existe en effet une Stéfanie, avec ses personnages, ses lieux - Trieste -, son histoire. On la retrouve dans les entrées 13 et 24 . 
la suite du Faux Journal et dans son œuvre. Cette entrée en matière par les voies du confessionnal où s'exhibe un discours provocateur (entre incestes et homosexualité) et sacrilège, joue sur deux tableaux. Le premier est celui de la condamnation de la confession en ce qu'elle a de faux. Le second est l'entrée en fiction du journal.

Après cet exercice de la confession, Jude Stéfan prend le soin de se dépeindre deux entrées plus loin, au moment choisi de «ce mois de purifications ou février » :

[...] pendant que j'y pense, je suis brun, l'air de me ficher du monde ; j'ai connu trente-six femmes, en ai aimé violemment, dévotieusement, quatre ; je porte des habits sur ma peau et mes os, marche debout, et si je rencontrais mon double - qui me hante, que je cherche - me haïrais fort ; dans quelque temps, irrévocablement, je n'aurais jamais vécu, ce qui prouve bien que j'ai le tort de me prendre entre-temps pour moi-même, sans âge, sans identité, la vie m'est un piège, une trappe cauchemardesque. Cependant je n'en pleure plus, me tiens coi, sec, les dents serrées.

Le Faux Journal oscille entre la radicalité du moi unique et l'appartenance à une communauté faite de « semblables 13 ». Pour se diluer et ne pas se diluer dans cette masse humaine, Jude Stéfan renforce la déréliction de ses notations. Ainsi les anecdotes qui concourent à dresser son autoportrait croquent-elles un être lâche (9, à l'hôpital) ; violent (2, bagarre avec un « type »; 24 , tentative pour en provoquer une), délibérément injurieux (5) et sans pitié. L'épisode de la rencontre du cul-de-jatte et de sa femme à la poste, supprimé par l'éditeur, a certainement été jugé de mauvais goût, mais c'est ce genre d'anecdote qui a pour rôle de surprendre, de remettre radicalement en place soi et les autres. Le narrateur, lui, vient de découvrir qu'il y avait « de la larve en [lui] »... À d'autres occasions, humour et autodérision forcent le trait : le tableau, alors sans limite, se veut déniaisant pour le lecteur qui nourrirait encore espoir et illusion sur autrui et sur lui-même. Le journal se plaît - et se complaît - à aller aussi loin qu'il le peut.

Cette lecture-thérapie a pourtant ses moments de grâce : si toutes les scènes d'amour n'embellissent pas le tableau, du moins permettent-elles que surgisse la complicité humaine avec la bonne, avec la chienne. Cela dit, l'amour ne subit aucun traitement de faveur : il s'en sort juste un peu mieux que le reste, aux côtés de l'attachement à la lecture du dictionnaire14.

Sans complaisance, la première évocation de la bonne par et dans l'accouplement brut, est par la suite plus nuancée. La voici dotée d'un prénom, Évelyne, d'une identité, d'un âge (9, « Tel 23 février de l'inexplicable présence »). Elle est tantôt bonne, tantôt servante, souvent associée aux promenades et déplacements, au restaurant, à la poste. Elle devient complice (20) lors de l'escapade hors de l'hôpital et surtout à la fin, pour la veillée mortuaire et l'enterrement de la chienne, tendrement aimée. Or justement, une parenté les lie dans les mots : la femme, chienne, au milieu des chiennes, c'est la formule que délivre la «Pensée » $\left(\mathrm{n}^{\circ} 17\right)$ que l'éditeur élimine du texte :

14. Pensée. Celle qui aurait le dessous de ta langue de la grosse S., la pleine pâte de celle de Solange T., le buste émerveillant de la blonde, les lèvres épaisses de la rousse, les seins de la juive, le connil de Mado, les yeux brûlants de la jeune garce, le sourire encore de la vénitienne au jasmin, la grâce de mon ex-femme, les doigts de C. Z., le port de Diane L., le fessier princier de Denise, l'entrecuisses de Germaine, les jambes de la poissonnière, tout cela à idolâtrer, arriverais-je enfin à l'aimer?

Un jour, déchiré par ces chiennes!

L'effet catalogue est atteint et rejoint les « litanies du scribe », autre genre pratiqué par Jude Stéfan, bien présentes dans ce texte, notamment dans le passage suivant qui liste « les lieux où (il peut) aller, en cas de crise ». Le sommaire qui dépersonnalise, tronçonne, marque la volonté de faire le tour de soi, de ses goûts, de ses tares plus que de ses vertus. Le goût appuyé pour tout ce que la société réprouve et partant, pour tous les discours mis-ogynes/ anthropes ${ }^{15}$, répond à une logique de l'expulsion de soi.

13. Entrée 5, « Jour de Vénus, 26 » : Un semblable s'est trompé d’imperméable au restaurant ».

14. Lecture toujours mentionnée dans des parenthèses comme pour signaler au lecteur $(3,7,18,39)$ son importance pour le futur auteur d'un autre dictionnaire, Pandectes ou le Neveu de Bayle, publié en 2008 (Paris, Gallimard).

15. On songe également à l'aveu d'Edmond de Goncourt, dans son journal, en 1891 : «mon journal n'a de valeur que par sa malveillance et je n'ai donné des gens de mon temps que des images grotesques... » (Journal 1887-1896, Paris, Robert Laffont, t. III, 22 février 1891, p. 547). 


\section{Expulsion de soi}

Qui dit «Pensée » peut ajouter «pari ». Il nous semble que ce qui apparente le Faux Journal à la littérature des moralistes, dans son étalage de déréliction, réside dans ce pari que fait Jude Stéfan d'expulser tout son être. Une entrée comme celle de "Saint Fidèle » qui commence par une prière, ne quitte pas le terrain de l'outrage mais rend manifeste cette attitude. Toutefois, pour éviter tout sérieux excessif et toute grandiloquence trompeuse, Jude Stéfan recourt à une image concrète : celle du ver solitaire dont il se délivre à l'extrême fin de son Faux Journal :

J'ai expulsé le ver. Quitté mon dernier compagnon de viscères. Comme on règle ses dettes avant de disparaître. Par propreté.

Cette expulsion, consécutive à la mort de la chienne, est suivie du départ de la « jeune bonne » et sonne alors le glas du journal. L'image du ver - « solitaire, lui aussi » (36) - résume donc le texte, en une sorte d'épitapheStéfan.

\section{Épilogue : retour au texte publié}

Ce parcours dans le texte premier du « Faux Journal » montre combien, par ses thématiques et sa construction, il perd de son intégrité et de sa substance à être "tronqué », comme le note J. S. sur la page de titre. Si le choix du titre - c'est à l'éditeur que l'on doit l'inversion de l'ordre primitif du titre et du sous-titre (Une vie de bran/ Faux Journal) - est finalement repris pour la « suite » publiée, le texte lui-même devient moins provocateur, moins fort littérairement. Avec quatorze suppressions sur les quarante et une entrées offertes, février et avril perdent leur place réelle dans l'année stéfanienne. Et le personnage, qui creuse ici sa tombe comme lui en vient l'idée « Jour de grâce, 18 » (7) puisqu'il « inexiste » (15), perd, lui, un peu de chair, d'os et d'humeurs.

Parallèlement, dans l'ensemble du volume collectif publié, «Faux Journal » trouve des échos multiples. Par les affinités électives qui unissent son auteur à des écrivains qui lui sont proches, frères ou modèles parmi lesquels Cioran, Georges Perros, Lorand Gaspar. Par les textes de réflexion théorique - en italique dans le volume - qui problématisent ce que «Faux Journal» met en œuvre. Il s'agit du lien unissant l'écriture et la mort qui renvoie à la réflexion de Maurice Blanchot évoquée, en fin de volume, par Alain Clerval ${ }^{16}$. Ainsi ne peut-on pas dire que ce texte n'ait pas ici sa place ni que la version éditoriale, avec ses amputations, en subvertisse le sens.

Notre étude comporte ses limites : nous n'avons pu examiner l'ensemble du processus de création. Il eût fallu, pour cela, avoir accès aux carnets de l'écrivain qui ont nourri le texte, ce qui n'est pas possible. Quant aux avant-textes du tapuscrit, on se souvient que Jude Stéfan dit les avoir détruits comme il le fait le plus souvent pour ses œuvres. Néanmoins, cet exemple permet de percevoir quelques strates de l'élaboration du journal. Il invite à lire autrement le titre. Le journal est bien faux puisque la spontanéité présumée de l'écriture diaristique est ici contredite par la recomposition stéfanienne. Il y aurait d'ailleurs beaucoup à dire sur cette spontanéité qui relève d'une vision schématique du journal et ignore l'écart entre écrire son journal et le publier. L'hostilité affichée de Jude Stéfan à l'égard du genre est surtout un rejet des journaux « de complaisance17 », un manifeste en faveur d'une écriture qui se place ailleurs, dans la vérité brute de l'être qui passe par une sorte d'autofiction. Lire ce premier Faux Journal aujourd'hui donne donc à voir l'horizon que le poète s'est très tôt fixé dans sa démarche d'écrivain, horizon auquel son œuvre est restée fidèle alors que l'horizon d'attente du lecteur, réel et supposé, a pu, quant à lui, évoluer au cours de ces trente-cinq années. Et l'on se plaît à imaginer quelle réception aurait aujourd'hui le vrai « Faux Journal » de 1975...

16. «Les limites du Journal intime», art. cit., p. 360-364.

17. Dans Épitomé (Éditions Le temps qu'il fait, 1993), Jude Stéfan consacre un article à Maine de Biran dont il distingue le journal des journaux où « se complaisent (les dragues, les bons mots, les racontars) » nombre d'auteurs. 
Marie-Françoise Lemonnier-Delpy est maître de conférences à l'université de Rouen, membre du CEREdI et de l'équipe «Genèse et Autobiographie » de l'ITEM. Elle a coédité, avec Catherine Viollet, Métamorphoses du journal personnel (AcademiaBruylant, 2006). Commissaire de l'exposition Jude Stéfan (Orbec, juin-juillet 2010) avec Marianne Alphant, elle prépare, avec elle, un volume de Mélanges consacrés à cet écrivain (2011). Spécialiste de Joseph Delteil (Joseph Delteil, une œeuvre épique au XXe siècle, IEO, 2007) elle travaille par ailleurs sur la question de l'épopée au $\mathrm{xx}^{\mathrm{e}}$ siècle, dans une perspective générique et génétique.

Marie-Françoise Lemonnier-Delpy, marie-francoise.lemonnier-delpy@univ-rouen.fr

\section{Résumés \\ Vrai « Faux Journal » de Jude Stéfan}

Le poète contemporain Jude Stéfan, né en 1930, répond, en 1975, à une commande de La Nouvelle Revue française pour un numéro spécial consacré aux « Journaux intimes inédits » qui couvre le xxe siècle. Il livre en effet un texte intitulé « Faux Journal » qui constitue son premier écrit diaristique. Ce texte donne à voir les choix d'écriture d'un écrivain qui affiche son hostilité à l'égard d'un genre qu'il juge «faux ». Or, à partir du tapuscrit auctorial revu par l'éditeur, consultable depuis peu, il est désormais possible d'étudier la genèse de ce singulier Faux Journal. Les corrections et recompositions effectuées par Jude Stéfan en constituent une première étape. L'examen de la genèse s'approfondit avec la comparaison entre le tapuscrit ainsi travaillé par le diariste et le texte publié. Cette deuxième étape donne à voir les interventions de l'éditeur. Celui-ci choisit de supprimer un certain nombre d'entrées pour ménager son lectorat en ne lui livrant pas un texte délibérément provocateur et outrancier. Mais la version non « tronquée » révèle un Faux Journal d'un rythme et d'une teneur différents, journal dans lequel se construit sous nos yeux un moi à la fois fictionnel et vrai. C'est l'épaisseur de ce texte inédit qu'analyse cet article.

The contemporary poet Jude Stéfan, born in 1930, responded to a commission from La Nouvelle Revue Française in 1975 for a special issue on twentieth-century "Unpublished Diaries". He produced a text titled "Faux Journal" (Fake Diary), his first diary writing. It shows the choices of a writer who flaunts his hostility toward a genre he considers "fake". Well, on the basis of the author's typescript revised by the publisher, a typescript only recently available, the genesis of this singular "Fake Diary" can now be studied. Jude Stéfan's corrections and rewriting are a first stage. The examination of the genesis is carried a step further by comparing the typescript reworked by the diarist to the published text. This second stage shows the publisher's interventions: he chose to suppress a certain number of passages to spare his readers by not giving them a deliberately provocative and extreme text. But the "uncut" version reveals a "Fake Diary" with a different rhythm and tone, a diary in which a both fictional and true "Self" appears before our very eyes. This article studies the density of this unpublished text.

Der zeitgenössische Dichter Jude Stéfan, geboren 1930, antwortet 1975 auf einen Aufruf der Nouvelle Revue française, die dem Thema „unveröffentlichte Tagebücher“ eine Sondernummer widmet, die das zwanzigste Jahrhundert umfasst. Er liefert in der Tat einen Text mit dem Titel „Faux Journal“, der sein erstes diaristisches Schreiben darstellt. Dieser Text zeigt die Schreibweisen eines Schriftstellers, der seine Feindseligkeit gegenüber einem Genre exponiert, das er als „,faux“ beurteilt. Nun ist es fortan möglich, die Genese dieses eigentümlichen „Faux Journal" anhand des vom Herausgeber lektorierten, vom Autor daktylographierten Manuskripts zu studieren, das seit kurzem eingesehen werden kann. Die Korrekturen und Neufassungen durch Jude Stéfan stellen eine erste Etappe der Analyse dar. Die Untersuchung der Genese lässt sich durch den Vergleich zwischen dem von dem Diaristen auf diese Weise bearbeiteten Manuskript und dem publizierten Text vertiefen. Diese zweite Etappe zeigt die Eingriffe durch den Herausgeber. Dieser entscheidet sich dafür, eine gewisse Anzahl von Einträgen zu streichen, und dadurch seine Leserschaft zu schonen, der er keinen absichtlich provokativen und maßlosen Text liefert. Die ungekürzte Version offenbart jedoch ein „Faux Journal“ von sehr unterschiedlichem Rhythmus und Wortlaut, ein Tagebuch, in dem sich vor unseren Augen ein Ich konstruiert, das zugleich fiktional und wahr ist. Dieser Artikel untersucht diese Dichte des unveröffentlichten Textes.

E1 poeta contemporáneo Jude Stéfan, nacido en 1930, responde en 1975 a un pedido de La Nouvelle Revue française para un número especial dedicado a los "Diarios íntimos inéditos" a lo largo del siglo $\mathrm{XX}$, entregando un texto titulado "Diario falso", que constituye su primer escrito de este tipo. Este texto revela las elecciones de escritura de un escritor que manifiesta su hostilidad con respecto a un género que considera "falso". Ahora bien, a partir del dactiloscrito del autor revisado por el editor, dactiloscrito consultable desde hace poco tiempo, se puede actualmente estudiar la génesis de ese singular "Diario falso". Las correcciones y reescrituras efectuadas por Jude Stéfan constituyen una primera etapa. La comparación entre este dactiloscrito pergeñado por el autor y el texto publicado permite profundizar el estudio de la génesis. Esta segunda etapa pone de relieve las intervenciones del editor, quien decide suprimir una cierta cantidad de entradas para preservar al lector de pasajes deliberadamente provocadores y excesivos. Pero la versión no "amputada" pone al descubierto un "Diario falso" con ritmo y contenido diferentes, diario en el cual vemos construirse un yo ficcional y verdadero a la vez. Este artículo analiza la densidad de ese texto inédito. 
O poeta contemporâneo Jude Stéfan, nascido em 1930, correspondeu, em 1975, a uma encomenda de La Nouvelle Revue française para um número especial consagrado a "diários íntimos inéditos", cobrindo o século XX. A sua entrega consistiu em um texto intitulado "Faux Journal", que constituiu o seu primeiro trabalho diarístico. O texto deixa ver as escolhas de escrita de um escritor que é hostil em relação a um género que considera "falso". Ora, a partir do dactiloscrito autoral revisto pelo editor, agora consultável, torna-se possível estudar a génese desse singular "Faux Journal". As correç̧ões e recomposições efectuadas por Jude Stéfan constituem uma primeira etapa. O exame da génese aprofunda-se com a comparação entre o dactiloscrito assim trabalhado pelo diarista e o texto publicado. Esta segunda etapa revela as intervenções do editor, que opta por suprimir diversas entradas, para poupar o leitor a um texto deliberadamente provocador e radical. Mas a versão não "truncada" revela um "Faux Journal" de ritmo e teor diferentes, um diário em que se constrói sob os nossos olhos um eu tanto imaginário como verdadeiro. É à densidade deste texto inédito que se dedica este artigo.
Il poeta contemporaneo Jude Stéfan, nato nel 1930, risponde nel 1975 a una richiesta della Nouvelle Revue française per un numero speciale sui “Diari personali inediti” del XX secolo. In realtà egli consegna un testo intitolato "Falso diario" che costituisce il suo primo scritto diaristico. Questo testo mostra le scelte di scrittura di un autore che non nasconde la sua ostilità riguardo un genere che giudica "falso". A partire da un dattiloscritto - da poco disponibile - dell'autore rivisto dall'editore, è ora possibile studiare la genesi di questo singolare "Falso diario". Le correzioni e ricomposizioni effettuate da Jude Stéfan ne costituiscono una prima tappa. L'esame della genesi si approfondisce con la comparazione tra il dattiloscritto così elaborato dal diarista e il testo pubblicato. Questa seconda tappa mostra gli interventi dell'editore. Costui sceglie di sopprimere un certo numero di passi per proteggere il lettore e non proporgli un testo deliberatamente provocatorio e oltraggioso. Ma la versione non "troncata" rivela un "Falso diario" dal ritmo e dal tenore differenti, diario nel quale sotto i nostri occhi si costruisce un io allo stesso tempo immaginario e vero. Questo articolo analizza le stratificazioni di questo testo inedito. 